\title{
Markarian and Seyfert Galaxies
}

\author{
E.Ye. Khachikian
}

Byurakan Astrophysical Observatory, Byurakan, 378433, Armenia

\section{Historical Perspective}

Since this symposium is devoted to the $90^{\text {th }}$ anniversary of Prof. Ambartsumian's birthday I would like to touch briefly upon some historical aspects of $\mathrm{V}$. Ambartsumian's ideas on activity of nuclei of galaxies.

Modern extragalactic astronomy achieved such high success that we have reached almost the stage of our Universe when it was only a couple of billion years old. It is very strange that some 75 years ago (an instant compared with the supposed age of the Universe) we had still no certain idea about the existence of remote galaxies. But already 10-15 years after discovering galaxies, it was a common idea that they were thoroughly formed steady systems with a rich past and no prospect of radical changes in the future. Except for classification of form of galaxies and investigation of their general photometry and colourimetry, nobody supposed that the most important part of galaxies is their central part. The role of nuclei in the evolution of galaxies was manifestly underestimated.

V. Ambartsumian was the first to pay particular attention to the significance of galactic nuclei. As was said figuratively by A. Sandage, "No astronomers would deny today, that mystery indeed surrounds the nuclei of galaxies, and Victor Ambartsumian was the first who understood what a rich reward is contained in this treasury".

In the second half of the 50's a new phase of extragalactic exploration set in, when the new concept of the basic role of the nuclei of galaxies in their life and evolution had been advanced by Ambartsumian (1956a,1956b, 1956c, 1958a, 1958b, 1961a, 1961b, 1962, 1968).

Of course the origin of this concept was not unfounded. It was preceded by a number of wonderful discoveries resulting in the revision of our notions on the world of galaxies.

In the first place was the identification of the powerful radio source Cygnus $A$ by Baade and Minkowski with a galaxy containing two nuclei (Baade \& Minkowski, 1954).

A similar picture was observed also in the radiosource Perseus A (NGC 1275). The role of two papers was also significant: Haro(1956) discovered 44 galaxies, unusually blue in colour; and, specially, in 1943 Seyfert's paper, now regarded as classical, should be singled out for mention.

The galaxies which he investigated are distinguished by the high luminosity of their nuclei, and, more importantly, by the width of the Balmer emission lines. The great width of the emission lines indicates that the turbulent motions of gas clouds in the nuclei of those galaxies, subsequently termed "Seyfert", at times attain a velocity of over $3000 \mathrm{~km} / \mathrm{s}$. Now it seems quite strange and surprising 
that this very important paper of Seyfert was not duly taken into account in the succeeding twenty years or so. It was only after Ambartsumian's idea concerning the activity of nuclei of galaxies had been made public that the astrophysicists returned to that paper, and a regular study of the Seyfert galaxies was started. On the basis of analysing these facts, Ambartsumian came to the idea of activity of nuclei of galaxies, which manifests itself mainly in the following forms :

1. Outflow of ordinary gas matter (in form of jets or clouds ) from the nuclear region at velocity of up to hundreds of $\mathrm{km} / \mathrm{s}$.

2. Continuous emission of flux of relativistic particles or other agents producing high energy particles, as a result of which a radio halo may form around the nucleus.

3. Eruptive ejections of gas matter (M 82 type).

4. Eruptive ejections of concentrations of relativistic plasma (NGC 4486, 5128 , etc.).

5. Ejection of compact blue condensations with an absolute magnitude of the order of luminosity of dwarf galaxies (NGC 3561, IC 1182). Here the division of the nucleus into two or more comparable components is also presumed, initiating the formation of multiple galaxies.

The presence of one or several of these phenomena allows us to call a galaxy active.At present a number of types of objects are considered as active: radio galaxies, QSOs, Seyfert galaxies, Lacertides, UV-excess galaxies, Blazars, Liners.

Let us come back to the phenomenon of radiogalaxies. Baade and Minkowski explained this phenomenon as a result of accidental collision of two galaxies. V.A. Ambartsumian was the first and only astronomer who in his works (1956b, 1956c, 1958a, 1961b, 1962), convincingly showed that in the case of radio-galaxies we have not a collision, but just activity of nuclei of galaxies, which brings ejection of matter from the nucleus, and in some cases as a result of this activity a radio-galaxy is originated.

I am lucky that I had the chance in 1961 in Berkeley to be present at the XI General Assembly of IAU, where V.Ambartsumian gave a public talk on the idea of activity of galaxies. The interest in his talk was so high that the conference hall and corridors were full of people (even outside, where microphones had been mounted). During this Assembly he was elected as President of the IAU.

It was really a revolutionary and extraordinary idea. It is said that in the Solvey conference in 1958 W.Baade even accused him of idealism and noted that for a scientist from the Soviet Union to speak about ejection and activity of nuclei of galaxies looked very strange. But just a couple of years later American astronomers Sandage and Lynds (1963) discovered the explosion in the center of the galaxy M82. Almost the same time in 1963 QSOs were discoverd. Actually, as Ambartsumian noted,they were just the naked active nuclei, which radiate an unusually high quantity of energy, highest among known cosmic objects. Now we know that many QSO are really surrounded by a stellar population. Thus, Ambartsumian's idea has been confirmed at first by observations of American astronomers. 
A new stage in the extragalactic field had been started: the era of active galaxies. The majority of large observatories over the world started to find new active galaxies (AG).By the leading of V.Ambartsumian the Byurakan observatory also engaged itself in activities aimed at discovering galaxies with active nuclei. Ambartsumian and Shahbazian (1954) were the first to show the existence of blue ejections and condensations associated with contiguous active elliptical galaxies. Subsequently Stockton (1968) showed that those objects are in fact associated with galaxies and display emission spectra similar to the associations.

Then on the initiative of V.A.Ambartsumian, B.E.Markarian started in Byurakan in the mid-sixties observations of the sky with a view to detecting galaxies with anomalous spectra, using the 40" Schmidt telescope with an objective prism of the same diameter.

\section{The First Byurakan Survey}

The first Byurakan survey (FBS) is the most famous work done with this telescope. More than 2000 photographic plates covering about 17000 square degrees of sky were obtained. Each plate contains low dispersion spectra $(2500 \mathrm{~A} / \mathrm{mm}$ near $H_{\beta}$ ) of more than 15000 objects. As a result, 1500 galaxies with strong UV-excess were discovered. In 1978 Markarian with his co-workers began the Second Byurakan Survey (SBS). The limiting magnitude of objects increased from $17^{m}$ (for FBS) up to about $19^{m} .5$ for SBS. The SBS covers about 1000 square degrees.

I was lucky to be the first to observe almost all galaxies from the first Markarian list of UV-galaxies with the largest optical telescopes of the USA. I would like to emphasize once more that the detailed spectral investigations of these objects indicated (Khachikian 1968, Weedman \& Khachikian 1968, 1969) that over $85 \%$ of them have emission lines, their intensity being directly dependent on the value of UV-excess. One can conclude that the presence of a strong ultraviolet continuum is closely associated with the formation of the emission spectrum and the more intense the continuous spectrum in the visible ultraviolet is, the more intense are the emission lines (see Fig 11.6 in Osterbrock, 1989).

It became also evident that the spectra of those objects differ, nevertheless, essentially from each other as to the excitation degree of the emission lines and their widths. Moreover, they turned out to differ sharply in morphological characteristics as well: one can come across the blue galaxies of Haro, the compact galaxies of Zwicky, the $\mathrm{N}$ type galaxies, spiral and irregular galaxies among the Markarian objects. Quite important is the discovery of the Seyfert galaxies and quasars among those objects. As far back as $1968 \mathrm{I}$ also demonstrated that on the basis of slitspectra, Markarian galaxies can be classified in five groups(Khachikian 1968):

1. Narrow line spectra both in emission and absorption.

2. Narrow, strong emission lines only.

3. Strong and diffuse emission lines; [0III] lines much stronger than the hydrogen lines ( Seyfert type 2 ). 
4. Very broad hydrogen lines,narrow forbidden lines (Seyfert type 1).

5. No strong emission lines (BL Lac type).

These results were presented at the first international conference on Seyfert galaxies and related objects in 1968 (Tuscon, Arizona, USA), where I called these objects "Markarian galaxies". Further spectral investigations of Markarian galaxies from both Byurakan Surveys have been carried out intensively in Byurakan and in many other observatories.

These searches show that among Markarian objects there are representives of all forms of activity predicted by V.Ambartsumian: QSOs, Seyfert galaxies, BL Lac objects, galaxies with jets, blue compact galaxies, double nuclei galaxies and so on. But the most important is, as was shown by Weedman and Khachikian (1971a), that $10 \%$ of Markarian galaxies turned out to be Seyfert galaxies.

The number of Seyfert type galaxies was extremely increased thanks to study of Markarian objects. In the original paper of Seyfert there are only 6 galaxies of Seyfert type. But now more than 1000 of these type of galaxies are known!

On the base of detailed spectral investigations of a number of Seyfert type galaxies Weedman and Khachikian (1971a) have shown that Seyfert galaxies clearly are divided into two types:

1. Galaxies with very broad hydrogen lines, and narrow forbidden lines (Seyfert type 1).

2. Galaxies with very broad both hydrogen and forbidden lines (Seyfert type 2).

There is also quite precise difference in relative intensities of some emission lines. In table 1 the relative intensities and equivalent widths of lines of typical Sy galaxies from the Markarian list are presented.

So, the second difference of Sy1 and Sy2 is the intensity ratio of emission lines $[O I I I]$ to $H_{\beta}$, which is less than unity for Sy1 and more than 10 for Sy2.

Therefore it is very strange sometimes to read in articles about narrow line Sy galaxies. There is no Sy galaxy with narrow lines! I would like to dwell here upon three subjects: a) morphology of Markarian and Seyfert galaxies; b) double nuclei $A G ; c)$ variability in the spectrum of $A G$.

As in this Symposium are presented many articles on Markarian and Seyfert galaxies in the range of radio, IR, UV, FUV, X- and Gamma ray, I shall concentrate on the optical properties of these objects.

a).The detailed morphological description of Markarian and Seyfert galaxies has been done by Khachikian (1987). It was shown that the activity is not correlated with the morphology of the whole galaxy: most important is the structure of the central part of galaxies. The majority of AG according to morphology of the central part can be divided into the following groups: i) starlike galaxies; ii) galaxies with starlike nucleus (in general spirals); iii) double nuclei galaxies; iv) multinuclei galaxies; v) galaxies with bulges. Some AG show jets starting from the nucleus. Representatives of these groups are shown in Fig1-Fig6 in Khachikian (1987). 
Table 1. From Weedman \& Khachikian (1969)

\begin{tabular}{|c|c|c|c|c|c|c|c|c|c|}
\hline & & \multicolumn{8}{|c|}{ Markarian numbers } \\
\hline & & \multicolumn{4}{|c|}{ Sy2 } & \multicolumn{4}{|c|}{ Sy1 } \\
\hline ion & $\lambda$ & 1 & 3 & 6 & 34 & 9 & 10 & 42 & 52 \\
\hline [S II] & $6717+$ & 3.30 & 6.11 & 0.62 & 2.85 & - & - & - & 1.29 \\
\hline & 6731 & - & - & - & - & - & - & - & - \\
\hline $\begin{array}{l}[N I I]] \\
H_{\alpha}\end{array}$ & $\begin{array}{l}6583+ \\
6562+\end{array}$ & 11.8 & 17.9 & 9.33 & 9.85 & 4.61 & 5.20 & 5.66 & 9.41 \\
\hline $\begin{array}{l}{[N I I]} \\
{[O I I I]}\end{array}$ & $\begin{array}{l}6548 \\
5007+ \\
4959\end{array}$ & 11.4 & 15.5 & 2.53 & 15.0 & 0.58 & 0.83 & 0.32 & 0.91 \\
\hline$H_{\beta}$ & 4861 & 1.0 & 1.0 & 1.0 & 1.0 & 1.0 & 1.0 & 1.0 & 1.0 \\
\hline He II & 4686 & - & 0.25 & - & - & - & 0.19 & - & - \\
\hline OIII & $\begin{array}{l}4363+ \\
4340\end{array}$ & 0.68 & 0.89 & - & 0.77 & 0.54 & 0.57 & 0.48 & 0.48 \\
\hline$[\mathrm{NeIII}]$ & 3869 & 1.08 & 1.34 & 0.16 & 1.57 & - & 0.17 & - & - \\
\hline$[O I I]$ & $\begin{array}{l}3726+ \\
3729\end{array}$ & 1.87 & 2.98 & 0.37 & 3.54 & - & 0.24 & - & 1.95 \\
\hline $\mathrm{H}_{\beta} / 10^{40} \mathrm{erg} / \mathrm{s}$ & & 2.1 & 5.8 & 18.6 & 14.2 & 72.5 & 36.2 & 2.8 & 1.1 \\
\hline & & \multicolumn{8}{|c|}{ equivalent widths } \\
\hline$[S I I]$ & $\begin{array}{l}6717+ \\
6731\end{array}$ & 50 & 92 & 16 & 44 & - & - & - & 33 \\
\hline $\begin{array}{l}{[N I I]} \\
H_{\alpha} \\
{[N I I]}\end{array}$ & $\begin{array}{l}6583+ \\
6562+ \\
6548\end{array}$ & 177 & 425 & 236 & 128 & 177 & 240 & 112 & 190 \\
\hline$[O I I I]$ & $\begin{array}{l}5007+ \\
4959\end{array}$ & 217 & 840 & 135 & 276 & 35 & 56 & 7 & 20 \\
\hline$H_{\beta}$ & 4861 & 17 & 36 & 43 & 21 & 59 & 61 & 21 & 19 \\
\hline He II & 4686 & - & 8 & - & - & - & 10 & - & - \\
\hline $\begin{array}{l}{[O I I I]} \\
H_{\alpha}\end{array}$ & $\begin{array}{l}4363+ \\
4340\end{array}$ & 11 & 41 & - & 15 & 23 & 27 & 8 & 8 \\
\hline$[N e I I I]$ & 3869 & 24 & 88 & 7 & 37 & - & 8 & - & - \\
\hline$[O I I]$ & $\begin{array}{l}3726+ \\
3729\end{array}$ & 41 & - & 15 & 78 & - & 11 & - & 31 \\
\hline
\end{tabular}




\section{Galaxies with Multiple Nuclei}

As it was mentioned above, the radio galaxy Cygnus $\mathrm{A}$ has two nuclei. It is interesting to note that the majority of AG turn out to be double nuclei. It is necessary to stress that in addition to double nuclei there are galaxies with three and more nuclei( or nuclear type formations). It is known also that each of the nuclei of double nucleus galaxies can themselves consist of two components ( Mark.273, Knapen et al.1998). Therefore the opinion concerning the nature of double nucleus AG is relevant to the multinuclei AG as well. The existence of galaxies with two condensations in the centre having Seyfert type spectra ( Mark.266, 463, 673, 789) is the most definite evidence of the possibility of galaxies with double nuclei in general. However, double nuclei galaxies are not an unusual phenomenon among AG. They are particularly common among UV galaxies. In Table 2 are some physical parameters: the apparent and absolute photographic magnitudes of the components of the nucleus, distance between components in arcseconds and kiloparsecs $(\mathrm{H}=75 \mathrm{~km} / \mathrm{s} . \mathrm{Mpc})$ and differences of their radial velocities for six AG with double nuclei are presented.

\begin{tabular}{rrrrrr} 
Table 2. From Khachikian $(1987)$ \\
\cline { 2 - 7 } Mark.No & $\mathrm{m}(\mathrm{pg})$ & $\mathrm{M}(\mathrm{pg})$ & $\mathrm{d}^{\prime \prime}$ & $\mathrm{d}(\mathrm{kpc})$ & $\mathrm{v}(\mathrm{km} / \mathrm{s})$ \\
\hline $266 \mathrm{a}$ & 17.5 & -17.8 & 12 & 6.5 & 127 \\
$\mathrm{~b}$ & 17.8 & -17.5 & & & \\
$273 \mathrm{a}$ & 17.5 & -18.4 & 4.3 & 3.2 & - \\
$\mathrm{b}$ & 18.2 & -17.7 & & & \\
$463 \mathrm{a}$ & 17.0 & -19.5 & 4.5 & 4.3 & 50 \\
$\mathrm{~b}$ & 17.2 & -19.3 & & & \\
$673 \mathrm{a}$ & 16.2 & -19.6 & 5.3 & 3.7 & 166 \\
$\mathrm{~b}$ & 16.2 & -19.6 & & & \\
$739 \mathrm{a}$ & 16.2 & -19.1 & 6.6 & 3.8 & 85 \\
$\mathrm{~b}$ & 17.0 & -18.3 & & & \\
$789 \mathrm{a}$ & 16.0 & -19.5 & 4.1 & 2.5 & 2 \\
$\mathrm{~b}$ & 18.0 & -17.5 & & & \\
\hline
\end{tabular}

From Table 3, where the sizes of nuclei, distance between them and difference of radial velocities of Sargent-Searle double nuclei objects are presented, one can see that for them we have the same picture.

The number of double and multi-nucleus AG is increasing all the time. Zwicky compact galaxies with emission spectrum, many radio galaxies, so-called isolated giant HII regions (Sargent \& Searle objects) or Superassociations (SA) are double nucleus objects. Among the galaxies from FBS more than 100 double nucleus galaxies were discovered. It is ridiculous that in the 60 's a few people spoke about the merging of galaxies, but now most of them explain any complicated nuclei of galaxies without hesitation as a merger. As is known now a lot of double and multiple nuclei galaxies, it seems that the Universe instead of red shifting is collapsing. Therefore I am sure that many of this type of nuclei are not a result of merging. No doubt, many of them (if not the majority) are real double nucleus galaxies. That is, they are probably not a result of merging or 
Table 3. From Shaver \& Chen (1985)

\begin{tabular}{rrrr}
\hline Object & $d^{\prime \prime}$ & $\mathrm{d}(\mathrm{kpc})$ & $\mathrm{v}(\mathrm{km} / \mathrm{s})$ \\
\hline $0128-531 \mathrm{~A}$ & 2.2 & 7.8 & 55 \\
$\mathrm{~B}$ & 0.9 & & \\
$1030+073 \mathrm{~A}$ & 1.1 & 4.3 & 25 \\
$\mathrm{~B}$ & 0.8 & & \\
$1247-043 \mathrm{~A}$ & 0.2 & 1.3 & 130 \\
$\mathrm{~B}$ & 0.3 & & \\
$1247-051 \mathrm{~A}$ & 0.05 & 0.7 & 20 \\
$\mathrm{~B}$ & 0.3 & & \\
\hline
\end{tabular}

interaction of two independent galaxies. Note that the following observational data are difficult to reconcile with the hypothesis of gravitational merging:

1. the discovery of Seyfert type double nuclei galaxies because of their rarity among galaxies (Khachikian, Petrosian, Sahakian 1978, 1980);

2. the discovery of twin-objects with quite identical spectra and morphology similar to two isolated SA being considered as one galaxy with double nuclei each of which is SA (Arp, Heidmann \& Khachikian 1974, Khachikian 1979);

3. the discovery of numerous double nuclei galaxies.

Aditionally, the excellent articles of Mazzarella and Boroson (1993) and Nordgren et al. (1995) support very much this idea.

It seems to me that one of the important current problems of $A G$ is the nature of their double and multiple nuclei: is it the result of merging of galaxies (two or more) or evolution of the central part of an individual galaxy. From the time of Kant and Laplace up to the present, the majority of theorists, as well as observers, believe that the Universe develops in a direction from concentrations of diffuse matter to the denser states. Perhaps V.Ambartsumian was the first who declared the opposite point of view. As far back as the end of the 40's, he stated the revolutionary idea that evolution in the Universe goes from the dense condition of matter to the rarefied one. It seems to me that many observational data speak in favour of this point of view. The existence of double and multi nuclei galaxies is a good As it was mentioned above one of the forms of activity is the ejection from the nuclei of active galaxies of isolated clouds with different contents. Further, both spectroscopic and morphological investigations show that the nuclei of some AG are variable and undergo irregular changes in brightness and in spectra. In the end of the 60's and the beginning of the 70's it became clear that in central parts of same AG take place physical processes leading to ejection of huge amounts of matter from the nucleus of AG. The small sizes of AG nuclei (AGN), about 10 arcsec, don't permit detecting such a new gas formation by means of direct observation in the near surroundings of them even in radio wavelengths. Therefore the only possibility for the investigation of these physical events is detailed spectrophotometrical observations with comparatively high dispersion. The most effective are investigations in the optical. 
The appearance of additional new emission components of Hydrogen lines in the spectrum of AGN first was discovered in 1969 by Khachikian and Weedman (1970,1971b). During one year (between February 1968 and January 1969) in the spectrum of Markarian 6, which was a Sy2 galaxy, new broad emission components of Hydrogen lines $H_{\alpha}, H_{\beta}$ and $H_{\gamma}$ were detected. Their blue-shift velocity corresponds to $3000 \mathrm{~km} / \mathrm{sec}$. In January 1970 the intensity of the $H_{\beta}$ component was equal to $50 \%$ of the basic $H_{\beta}$ line. These observations have been confirmed by many authors (Adams 1972, Notni et al. 1973, Adams \& Weedman 1975, Chuvaev 1991, Khachikian 1973, Khachikian et al.1982). It is known now many active objects with double Hydrogen line structure in the spectrum: $3 \mathrm{C}$ 390.3, NGC 1097, NGC 1566 (Lynds 1968, Pastoriza \& Gerola 1970, Alloin et al.1986). There are some models which have been suggested to explain this phenomenon (Khachikian \& Weedman 1971, Khachikian 1973, Pronik 1987, Zheng et al. 1990, Eraclous \& Halperin 1992, Chen et al 1989). All these models do not give a complete explanation of the phenomenon.In Ambartsumian et al. (1998) a new fairly simple model is suggested, which gives quantitative accordance with observational data. In Fig. 1 the schematic picture of the model is shown.

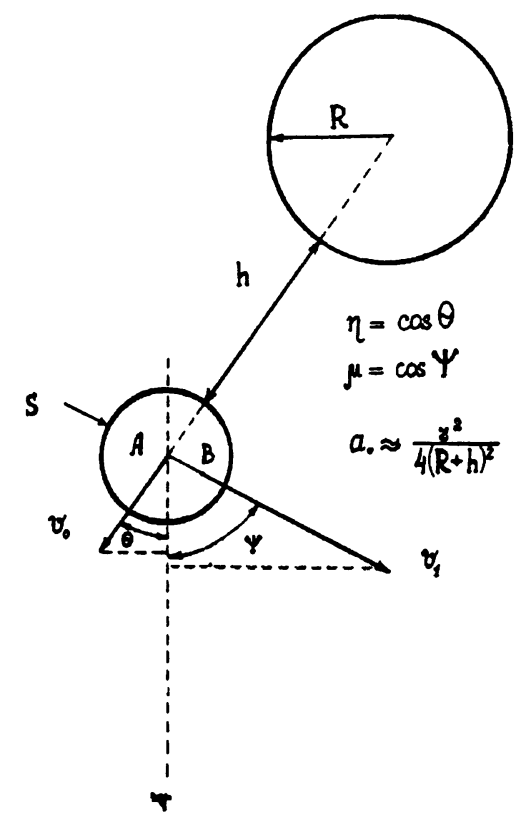

Figure 1.

From the nucleus of $A G$ a compact formation $S$ is ejected with the velocity Vo. At some distance from the surface of the AG it exploded. As a result of this explosion a globular gas cloud $\mathrm{S}$, mainly consisting of Hydrogen atoms, is formed. Similar to the situation in planetary nebula, the $L_{c}$-quanta of $\mathrm{AG}$ lead to ionization of Hydrogen atoms in $\mathrm{S}$. As a result of recombination and following cascade transitions the subordinate Hydrogen lines are formed. On the whole the additional components of subordinate lines arise. The shift of 
additional lines relative to that of the nucleus of $A G$ is explained by the speed of ejection of S from AGN. As for the widths of additional lines they depend on the velocity of expansion of S. The simple estimation presented in this work shows that comparable small size and mass of $S$ cloud and an acceptable value of speed of its expansion can explain the phenomenon.

The terms "active galaxies", "active nucleus", introduced by Ambartsumian, are now generally accepted in science, although some scientists (mostly younger one) have no idea about that. Therefore, his followers sometime have to remind us about the tremendous impact of V.Ambartsumian in science, in particular in extragalactic astronomy. Ambartsumian's idea on the activity of galaxies exerted tremendous influence on the further development of extragalactic astronomy and stimulated numerous studies in many observatories over the world. In the well-known Volume of the U.S. Academy of Sciences "The Heritage of Copernicus", commemorating the quinquecentennial of the birth of Copernicus, this Ambartsumian idea is considered as a Copernican type of revolutionary idea, which has changed our notion about the nature of galaxies.

\section{References}

Abramian, M. G., Fridman A. M., \& Khachikian E. Ye., 1994, In "The Cold Universe". Moriond Astrophysics Meeting, Les Arcs, France, p.357

Adams, T. F., 1972, ApJ, 172, L 101

Adams, T. F., \& Weedman, D. W., 1975, ApJ, 199, 19

Adams, T. F., 1972, ApJ, 172, L 101

Alloin, D., Potal, D., Fosbury, R. A., Freeman, K., Phillips, M. M. 1986, ApJ, 207, L 147

Ambartsumian, V. A., 1956a, Proceedings of V Conference on Problems of Cosmogony, Moscow.

Ambartsumian, V. A., 1956b, Izv. Acad. Sci. Arm. SSR, serie Phys. Math. Sci., 9,23

Ambartsumian, V. A., 1956c, Dokladi of AS of Armenia, 23, 161

Ambartsumian, V. A., 1958a, Izv. Acad. Sci. Arm. SSR, serie Phys. Math. Sci., 11,9

Ambartsumian, V. A., 1958b, "La structure et l'evolution de l'univers", Solvey Conference, p.241, Bruxelles, ed.by R.Stoops

Ambartsumian, V. A., 1961a, "The problems of extragalactic resurch",General Assamble of IAU, Berkeley

Ambartsumian, V. A., 1961b, AJ, 66, 536

Ambartsumian, V. A., 1962, The problems of Cosmogony, 8, 3

Ambartsumian, V. A., 1968, The Problems of Evolution of the Universe, Yerevan, p. 85

Ambartsumian, V. A., \& Shahbazian, R. K., 1957, C. R. Acad. Sci. Arm., 25, 185

Ambartsumian, V. A., Khachikian E. Ye., \& Yengibarian, N. B., Astrofizika, $1998,41,321$ 
Arp, H. C., Heidmann, J., \& Khachikian, E. Ye., 1974, Astrofizika, 10, 7

Baade, W., Minkovski, R., 1954, AJ, 119, 206

Chen, K., Halperin, J., \& Filippenko, A. V., 1989, ApJ, 339, 742

Chuvaev, K. K., 1991, Izv. Crimean. Astr. Observ., 83, 194

Eraclous, M., \& Halperin, J., 1992, in "Testing the AGN Paragdigm". Ed. by S. Holt

Haro, G., 1956, Bol. obs. Tonantzintla, 14, 8

Khachikian, E. Ye., 1968, AJ, 73, 891

Khachikian, E. Ye., 1973, Astrofizika, 9, 139

Khachikian, E. Ye., 1979, "Stars and Star Systems", Uppsala, p.107

Khachikian, E. Ye., 1987, IAU Symp. No121. Ed. by Khachikian et al., Byurakan, p.65

Khachikian, E. Ye., Petrosian, A. R., \& Sahakian, K. A., 1978, Astrofizika,

Khachikian, E. Ye., Petrosian, A. R., \& Sahakian, K. A., 1980, Astrofizika, 16, 621

Khachikian, E. Ye., \& Weedman, D. W., 1970, Astron. Zircul. No591, 2. 14, 69

Khachikian, E. Ye., Popov, V. N., Yegiazarian, A. A., 1982, Astrofizika, 18, 541

Khachikian, E. Ye., \& Weedman, D. W., 1971, Astrofizika, 7, 389

Khachikian, E. Ye., \& Weedman, D. W., 1971, ApJ, 164, L109

Knapen, J. H., Laine, S., Yates, J. A., Robinson, A., Richards, A., M., Doyon R., \& Nadeau D., 1998, ApJ(in press)

Lynds, C. R., 1968, AJ, 73, 888

Lynds, C. R., \& Sandage, A. R., 1963, AJ, 137, 1005

Mazzarella, J. M., \& Boroson, T. A. 1993, ApJS,85, 27

Nordgren, T. E., Helou, G., Chengalur, J. N., Terzian, Y., \& Khachikian, E. Ye. 1995, ApJS, 99, 461

Notni, P., Khachikian, Ye. E., Butslov, M. M., \& Gevorkian, G. T., 1973, Astrofizika, 9, 39

Osterbrock, D., 1989, in "Astrophysics of Gaseous Nebulae and Active Galactice Nuclei"

Pronik, I. I., 1987, in IAU Symp. No 121. Ed. by Khachikian et al., Buyrakan, p. 169

Sargent, W. L. W., 1970, AJ, 159, 765

Seyfert, C., 1943, AJ, 97, 28

Shaver, P. A., Chen, J.-S., 1985, A\&A, 148, 443

Stockton, A., 1968, AJ, 73, 887

Weedman, D. W., \& Khachikian, E. Ye., 1968, Astrofizika, 4, 587

Weedman, D. W., \& Khachikian, E. Ye., 1969, Astrofizika, 5, 113

Zheng, W., Binette, L., Sulentic, J., 1990, ApJ, 365, 115 